\title{
Efecto de la temperatura y el tiempo sobre los indicadores de la papa (Solanum tuberosum) fermentada en estado sólido
}

\section{Effect of temperature and time on indicators of potato (Solanum tuberosum), fermented in solid state}

Fecha recepción: 12 de febrero de 2014 Fecha Aprobación: 9 de junio de 2014
Luis Miguel Borras-Sandoval' ${ }^{1}$, Arabel Elías Iglesias², Mónica Andrea Moyano-Bautista ${ }^{3}$

\section{Resumen}

El presente documento pretende evaluar el efecto que producen la temperatura y el tiempo de incubación en algunos indicadores fermentativos de papa sometida a fermentación en estado sólido. Se utilizó un diseño completamente aleatorio con arreglo factorial (tres tratamientos de temperatura $\left(20^{\circ}, 25^{\circ}\right.$ y $30^{\circ} \mathrm{C}$ ) y dos tratamiento de tiempo ( 24 y 48 horas), con tres repeticiones para cada uno. La papa fresca y picada se mezcló con un material secante, melaza, urea, microorganismos eficientes, premezcla mineral y sulfato de sodio y se dejó fermentar acorde con el tiempo y la temperatura, según tratamiento, en bolsas plásticas de un kilogramo de capacidad. El producto Fes-papa incubado a distintas temperaturas presentó modificaciones significativas $(\mathrm{P}<0.01)$ en los indicadores fermentativos evaluados. $\mathrm{El} \mathrm{pH}$ descendió constantemente desde el inicio (5.7) hasta terminar la evaluación $(\mathrm{pH} 4.4)$, a las 48 horas, independientemente de la temperatura de

\begin{abstract}
This paper evaluate the effect of the temperature and incubation time in some fermentative indicators of potato subjected to solid-state fermentation. We used a completely random design with factorial arrangement: three temperature treatments $\left(20^{\circ}\right.$, $25^{\circ}$ and $30{ }^{\circ} \mathrm{C}$ ) and two time treatments (24 and 48 hours), with three replicates for each one. We mixed fresh chopped potatoes with a drying agent, molasses, urea, effective microorganisms, mineral premix and sodium sulfate; and we placed the mixture in $1 \mathrm{Kg}$ plastic bags to ferment according to the time and temperature treatments. The Fespotato product incubated at different temperatures showed significant changes $(\mathrm{P}<0.01)$ in the evaluated fermentative indicators. The $\mathrm{pH}$ declined steadily since the beginning $(\mathrm{pH} \mathrm{5.7)}$ to the end of the assessment after 48 hours $(\mathrm{pH} 4.4)$ regardless of the incubation temperature. Similarly, the ammonia concentration $(\mathrm{NH} 3-\mathrm{N})$ and total volatile fatty
\end{abstract}

1. Ph.D. (c). Universidad Pedagógica y Tecnológica de Colombia (Tunja - Boyacá, Colombia).

2. Ph.D. Instituto de Ciencia Animal - ICA (Mayabeque, Cuba). aelias@ica.co.cu.

3. Profesional Independiente (Tunja - Boyacá, Colombia). 
incubación. Algo similar ocurrió con la concentración de amoniaco $(\mathrm{N}-\mathrm{NH} 3)$ y de ácidos grasos volátiles totales (AGVt), los cuales descendieron con el tiempo de fermentación; valores relacionados con el descenso del $\mathrm{pH}$. El contenido en materia seca (MS) aumentó (de 19.3 a $38.5 \%$ ) con el incremento en la temperatura a las 24 horas, mientras que a las 48 horas descendió (de 38.1 a 17.9\%). La Fes-papa es un proceso biotecnológico sencillo para aprovechar los tubérculos de los residuos de cosecha para generar un alimento energético-proteico que, acorde con los indicadores fermentativos y contenido en MS, pudiera ser empleado en la alimentación animal; lo cual, además, contrarrestaría la contaminación ambiental.

Palabras clave: Fermentación en estado sólido, Incubación, Contaminación ambiental, Papa, Biotecnología, Microorganismos eficientes. acids (AGVt) decreased with fermentation time related to the decrease of $\mathrm{pH}$. The dry matter (DM) content increased (19.3 to $38.5 \%$ ) with the raise in temperature at 24 hours, whereas it decreased at 48 hours (38.1 to $17.9 \%$ ). The Fes-papa is a simple biotechnological process that takes advantage of the tubers crop residues to generate an energy-protein food, which according to the fermentative and content in MS indicators could be used in animal feed in addition to counter environmental pollution.

Keywords: Solid State Fermentation, Incubation, Environmental Pollution, Potatoes, Biotechnology, Effective Microorganisms. 


\section{Introducción}

El cultivo de la papa (Solanum tuberosum) en Colombia representa, en promedio, el $32 \%$ de la producción de los cultivos transitorios; ocupó el cuarto lugar en la producción agropecuaria nacional en el 2011, después del cultivo de la caña azucarera, la caña panelera y el plátano, con 3.156.813 millones de toneladas y un área cosechada de 1.565.689 hectáreas $(1,2)$.

En las épocas del año en que se colecta la cosecha de papa, los precios del tubérculo descienden, debido al exceso de la oferta; esta circunstancia ha llevado a que tradicionalmente se pierdan cantidades considerables de la cosecha, debido a la poca utilidad económica que se obtiene al Ilevarla al mercado. Por otra parte, aun en condiciones de mercadeo normal existe una porción de la papa cosechada que por su tamaño reducido y daños físicos durante la recolección, no puede ser comercializada; tanto los excedentes como los desechos de la papa se han utilizado en la alimentación animal (3).

El tubérculo está constituido por, aproximadamente, 2\% de cáscara, 75\% a 85\% de parénquima vascular de almacenamiento y $14 \%$ a $20 \%$ de médula (4). La calidad de la papa es influenciada por las condiciones ambientales y de manejo agronómico $(4,5)$; se ha establecido que recién cosechada contiene entre $70 \%$ y $75 \%$ de humedad y entre $25 \%$ y $30 \%$ de materia seca (MS), de la cual, entre el $60 \%$ y el $80 \%$ es almidón (4); sus carbohidratos incluyen almidón, celulosa, glucosa y sacarosa. El contenido de proteína es análogo al de los cereales, con valores fluctuantes entre $6 \%$ y $12 \%(6)$, y se han identificado cerca de 21 aminoácidos como constituyentes normales en el tejido del tubérculo (4).

Históricamente, en las zonas de producción de Colombia se ha utilizado la papa para la alimentación animal, fundamentalmente en vacas en pastoreo, en forma fresca o ensilada $(7,8)$, con resultados satisfactorios; sin embargo, por sus características de altos tenores de carbohidratos amiláceos y bajos en fibra y proteína bruta (PB), se pudiera convertir en materia prima como fuente energética, en fermentación en estado sólido (FES), con la adición de urea (nitrógeno no proteico), y biotransformarla en un producto proteico- energético denominado Saccharina, según la tecnología desarrollada en el Instituto de Ciencia Animal de Cuba (9), a partir de la caña de azúcar, con mejores indicadores y digestibilidad que el producto fermentado (papa).

El objetivo del presente trabajo fue evaluar el efecto que produce la temperatura y el tiempo de incubación en algunos indicadores fermentativos de la papa fermentada en estado sólido.

\section{Materiales y Métodos}

Localización geográfica del área de estudio. La investigación se llevó a cabo en el Laboratorio de Nutrición Animal de la Universidad Pedagógica y Tecnológica de Colombia (UPTC), en el municipio de Tunja, departamento de Boyacá, Colombia.

Procedimiento experimental. En la preparación del producto FES-papa se empleó tubérculo completo de papa obtenida comercialmente, previamente limpio y picado finamente, y se añadieron los siguientes ingredientes y fuentes energéticas, según método descrito preestablecido (9) así: 90\% de papa, 5\% de material secante (salvado de trigo), 2\% de melaza, 1\% de urea, $1 \%$ de microorganismos eficientes, $0,5 \%$ de premezcla mineral y $0,5 \%$ de sulfato de sodio. Estos ingredientes se mezclaron hasta obtener una pasta homogénea, que denominamos producto Fes-papa; parte de este se dispuso para realizar los análisis de indicadores fermentativos del producto a la hora 0 y a temperatura ambiente, y el producto restante se distribuyó en bolsas plásticas selladas no herméticamente de $1 \mathrm{~kg}$ de capacidad, para incubar a diferentes temperaturas y periodos de tiempo, según tratamientos; cada bolsa representó una unidad experimental, con tres repeticiones cada una.

El producto se mantuvo a $20^{\circ} \mathrm{C}, 25^{\circ} \mathrm{C}$ y $30{ }^{\circ} \mathrm{C}$, respectivamente, en incubadoras independientes marca Memmert ${ }^{\circledR}$; pasadas 24 y 48 horas de incubación se procedió a tomar parte de cada una de las muestras del producto para análisis químicos.

Análisis químico. Se tomaron $5 \mathrm{~g}$ de muestra, se colocaron en un Erlenmeyer de $100 \mathrm{~mL}$ y se les adicionó $45 \mathrm{~mL}$ de agua destilada estéril (fresca). 
La preparación se agitó durante 30 minutos en un agitador eléctrico marca Adams ${ }^{\circledR}$ y posteriormente se obtuvo el filtrado mediante gasas estériles para medición del $\mathrm{pH}$ en un potenciómetro automático marca Okaton ${ }^{\circledR}$, tecnología desarrollada por Elias et al. (9), el nitrógeno amoniacal $(\% \mathrm{~N}-\mathrm{NH} 3)(10)$ y los ácidos grasos de cadena corta totales (AGVt) (11) y Materia Seca (MS) (12).

Diseño experimental. Se utilizó un diseño completamente aleatorio con arreglo factorial (tres tratamientos de temperatura y con dos horarios de muestreo), y tres repeticiones por tratamiento de la siguiente forma:

Tabla I. Tratamientos establecidos.

\begin{tabular}{|l|l|}
\hline Tratamiento 1 & $24 \mathrm{~h} *-20^{\circ} \mathrm{C}$ \\
\hline Tratamiento 2 & $24 \mathrm{~h}-25^{\circ} \mathrm{C}$ \\
\hline Tratamiento 3 & $24 \mathrm{~h}-30^{\circ} \mathrm{C}$ \\
\hline Tratamiento 4 & $48 \mathrm{~h}-20^{\circ} \mathrm{C}$ \\
\hline Tratamiento 5 & $48 \mathrm{~h}-25^{\circ} \mathrm{C}$ \\
\hline Tratamiento 6 & $48 \mathrm{~h}-30^{\circ} \mathrm{C}$ \\
\hline
\end{tabular}

* h: tiempo en horas

Los datos obtenidos se analizaron con las pruebas estadísticas ANOVA, con el fin de determinar diferencias entre tratamientos. Para la comparación de medidas se utilizó la prueba de Tukey, a través del programa SAS, versión 9.2 para Windows (13).

\section{Resultados y Discusión}

En la Tabla II se presentan los valores de $\mathrm{pH}$ de cada tratamiento.
Tabla II. Promedio de los valores de $\mathrm{pH}$ medidos en los diferentes tratamientos.

\begin{tabular}{|l|l|l|}
\hline \multicolumn{2}{|l|}{ TRATAMIENTO } & pH \\
\hline T0 & $\mathrm{O} \mathrm{h}$ & 5,7 \\
\hline T1 & $24 \mathrm{~h}-20^{\circ} \mathrm{C}$ & 5,1 \\
\hline T2 & $24 \mathrm{~h}-25^{\circ} \mathrm{C}$ & 4,6 \\
\hline T3 & $24 \mathrm{~h}-30^{\circ} \mathrm{C}$ & 4,3 \\
\hline T4 & $48 \mathrm{~h}-20^{\circ} \mathrm{C}$ & 4,5 \\
\hline T5 & $48 \mathrm{~h}-25^{\circ} \mathrm{C}$ & 4,4 \\
\hline T6 & $48 \mathrm{~h}-30^{\circ} \mathrm{C}$ & 4,4 \\
\hline
\end{tabular}

En este sentido, se produjo un descenso del $\mathrm{pH}$ en el tiempo, desde el inicio de la fermentación $(0 \mathrm{~h})$ hasta las $48 \mathrm{~h}$, independientemente de la temperatura de incubación durante el proceso de fermentación. Resultados similares fueron obtenidos por (14), al incrementar niveles de inclusión de tubérculos de yuca en FES de la caña de azúcar, con valores que oscilaron de 5 a 4.5, y por (9), quien obtuvo valores de 4.5 , o por debajo, a las $48 \mathrm{~h}$ al incluir diferentes fuentes de cereales o proteínas $(15,16,17)$. Este descenso pudo estar relacionado con la producción de ácido láctico, debido a la presencia de azúcares y almidones en la papa, y a la composición del inóculo, rico en bacterias lácticas. Otros (18) encontraron que en procesos de FES con mezclas de granos, residuos de cosecha $u$ otros ingredientes durante el catabolismo de los carbohidratos se producen aumentos considerables de ácido láctico, producto del gran crecimiento de bacterias lácticas.

Amoniaco (\%N-NH3). Como se aprecia en la Tabla III, el \%N-NH3 presentó un incremento en los tratamientos a las $24 \mathrm{~h}$, al aumentar la temperatura de $20^{\circ}$ a $25^{\circ}$ y $30{ }^{\circ} \mathrm{C}$, sin hallarse diferencias entre las dos últimas. A las $48 \mathrm{~h}$ de incubación, la concentración de $\mathrm{NH} 3$ descendió en todas las temperaturas de incubación, sin presentar diferencias significativas.

Tabla III. Promedios de cada una de las variables en los diferentes tratamientos.

\begin{tabular}{|c|c|c|c|c|c|c|c|}
\hline \multirow[t]{2}{*}{ VARIABLE } & \multicolumn{6}{|c|}{ TRATAMIENTOS } & \multirow[b]{2}{*}{ Sign } \\
\hline & $\begin{array}{ll}\mathrm{T} & 1 \\
24 \mathrm{~h}-20^{\circ} \mathrm{C}\end{array}$ & \begin{tabular}{|l} 
T2 \\
$24 h-25^{\circ} \mathrm{C}$
\end{tabular} & \begin{tabular}{|l} 
T3 \\
$24 h-30^{\circ} \mathrm{C}$
\end{tabular} & $\begin{array}{l}\mathrm{T} \\
48 \mathrm{~h}-20^{\circ} \mathrm{C}\end{array}$ & $\begin{array}{l}\mathrm{T} \\
48 \mathrm{~h}-25^{\circ} \mathrm{C}\end{array}$ & T6 48h-30 ${ }^{\circ} \mathrm{C}$ & \\
\hline Amoniaco (\%N-NH $)_{3}$ & $0.0052^{b}$ & $0.0092^{a}$ & $0.0090^{a}$ & $0.0047^{b}$ & $0.0054^{b}$ & $0.0033^{b}$ & $* *$ \\
\hline $\mathrm{AGV}(\mathrm{mEq} / \mathrm{l})$ & $0.6228^{b, c}$ & $5.7855^{\mathrm{a}}$ & $1.6297^{b}$ & $0^{c}$ & $0^{c}$ & $0^{c}$ & $* *$ \\
\hline Humedad (\%) & $80.7^{a}$ & $60.5^{b}$ & $61.4^{\mathrm{b}}$ & $61.8^{b}$ & $80.8^{a}$ & $82^{a}$ & $* *$ \\
\hline Materia seca (\%) & $19.3^{b}$ & $39.4^{\mathrm{a}}$ & $38.5^{\mathrm{a}}$ & $38.1^{a}$ & $19.1^{\mathrm{a}}$ & $17.9^{\mathrm{a}}$ & $* *$ \\
\hline
\end{tabular}

a, b, c, d Letras diferentes en la misma fila muestran diferencias estadísticas significativas. ${ }^{* *}(\mathrm{P}<0.01)$ 
La elevación del $\mathrm{N}-\mathrm{NH} 3$ en las primeras $24 \mathrm{~h}$ de incubación pudo estar relacionada con la implantación y actividad de microorganismos ureolíticos, como ha sido reportado por varios trabajos $(9,19,20)$; valores que coinciden con las concentraciones de $\mathrm{N}-\mathrm{NH} 3$ (21) en los que se adicionó Vitarfert (Microorganismos eficientes) a los desechos de caña de azúcar, y en Sacchasorgo y Sacchapullido con adición del 5\% de Vitafert (22) a temperatura de $25{ }^{\circ} \mathrm{C}$. Este comportamiento puede atribuirse a la utilización del N-NH3, así como de los azucares disponibles por los microorganismos que prevalecen en el producto, para la formación de su protoplasma celular $(9$, 22). Algo similar reportó (23) en un estudio de FES-bagacillo de arroz, elaborado con $14 \mathrm{t}$ de bagacillo de retorno, $1.5 \%$ de urea, $0.5 \%$ de sales minerales, $10 \%$ de pulidura de arroz y $15 \%$ de Saccharina como inóculo, el cual, después de 24 h de fermentación, se ensiló en bolsas de nailon durante $45 \mathrm{~d}$ y finalmente se realizó medición de parámetros.

Así mismo, se observó un comportamiento similar en la concentración de amoniaco en un trabajo realizado en FES de mezcla de caña y boniato con adición de urea del $1 \%(24)$, cantidad igual a la usada en el presente estudio. Los investigadores observaron valores de 6,4 y $1,66 \mathrm{mEq} / \mathrm{L}$ deamoniaco a las 0 y $48 \mathrm{~h}$ de fermentación, respectivamente, que demuestran la disminución de los valores de $\mathrm{NH} 3$ con el transcurso del tiempo; esto coincidió con una disminución significativa del $\mathrm{pH}(\mathrm{P}<$ 0.001 ) durante las primeras $48 \mathrm{~h}$; posteriormente ascendió de forma notable en aquellos tratamientos con mayor nivel de nitrógeno, según estos autores, lo que concuerda con el presente estudio, donde las mediciones se realizaron únicamente para las primeras 24 y $48 \mathrm{~h}$ de fermentación.

Cuando existe un $\mathrm{pH}$ bajo, como el medido para los tratamientos a las 24 y $48 \mathrm{~h}$ (Tabla II), el NH3 producido es retenido en el sustrato $(9,13,22$, 24). Este amoniaco, como compuesto, puede ser utilizado como fuente de nitrógeno por los microorganismos estabilizados durante el proceso de fermentación, y como resultado, la cantidad de biomasa microbiana presente en los sustratos fermentados se incrementa (25), disminuyendo la concentración amoniacal en el tiempo.

En cuanto a esto, se recalca que el crecimiento de los microorganismos desarrollados durante los procesos de fermentación en estado sólido no solo dependerá de los sustratos empleados, sino también de los factores biológicos y físicoquímicos, como la disponibilidad energética, la temperatura, el $\mathrm{pH}$, la aireación y el tiempo de incubación de la muestra $(26,28)$.

Ácidos grasos volátiles (AGVt). Los (AGVt) presentaron un incremento significativo $(\mathrm{P}<0.01)$ a las $24 \mathrm{~h}$ y $25{ }^{\circ} \mathrm{C}$ de fermentación, pero en los muestreos de las $48 \mathrm{~h}$ se hallaron valores definidos de $0 \mathrm{mEq} / \mathrm{L}$. La mayor concentración de AGVt en el tratamiento 2 durante el proceso de FES-papa se relaciona con otros experimentos (22), donde se ha empleado menos del $2 \%$ de urea en FES-caña de azúcar; la baja cantidad de urea $(1 \%)$ empleada en el presente trabajo puede haber limitado la disponibilidad del $\mathrm{NH} 4$ para los microorganismos.

Al considerar lo anterior, los microorganismos harían uso de los aminoácidos libres del protoplasma, entrando en fase de latencia con cúmulo interno de polisacáridos celulares (22), y fermentando los azúcares disponibles en el proceso de FES con producción de AGVt. Este proceso llevaría al agotamiento de los carbohidratos solubles disponibles para la actividad energética de la microbiota durante las primeras 24 horas de fermentación, por lo que disminuye la producción de metabolitos como los AGV (9), lo que se vio reflejado en los valores de $0 \mathrm{mEq} / \mathrm{l}$ a las $48 \mathrm{~h}$ de fermentación en el presente trabajo, ya que la amilasa que pudieran segregar los microorganismos establecidos en la FES es muy sensible en medios de $\mathrm{pH}$ bajos, como los obtenidos en el presente experimento, entre $4.4 \mathrm{y}$ 4.5 a las $48 \mathrm{~h}$, independientemente del tiempo de incubación $\left(20^{\circ}, 25^{\circ}\right.$ y $30^{\circ} \mathrm{C}$, ver Tabla II).

Con el efecto del tiempo, reporta (27) que la fermentación en FES-boniato no afectó la concentración de ácido láctico, y los menores valores de acético se encontraron a las $48 \mathrm{~h}$, al igual que en el presente trabajo, en relación con la producción de AGVt.

Los resultados de este trabajo en la disminución de la concentración de ácidos grasos volátiles en diferentes tiempos y temperaturas también coinciden con lo propuesto por (22) y (24), que durante el proceso de FES de desechos de caña de azúcar sugirieron que los microorganismos obtienen la energía de la oxidación de los 
carbohidratos (sacarosa, glucosa, fructosa y almidón) del sustrato y de las fuentes energéticas empleadas, con la producción final de energía en forma de ATP, de AGV, ácidos orgánicos, $\mathrm{CO}_{2}$ y $\mathrm{H}_{2} \mathrm{O}$. Estos productos de desecho de los microorganismos se convierten en el sustrato de otros; así, probablemente, en el presente estudio los AGV pudieron ser usados por las levaduras y las bacterias como elementos estructurales para la construcción de nuevas células que, finalmente, se convertirían en proteína.

Humedad (H) y materia seca (MS). Se han reportado (28) valores de humedad para la papa cruda del 75$76 \%$, y porcentajes de materia seca del $19.9 \%$ (7). En un estudio previo realizado (29) se determinó un porcentaje de $86,76 \%$ de $\mathrm{H}$ en la papa cruda, valor similar al obtenido para el producto FES-papa recién preparado en este trabajo, de $84,1 \% \mathrm{H}$ y $17,7 \%$ MS, lo que puede atribuirse a la adición de los microorganismos eficientes en estado líquido y a la urea diluida en agua destilada. Adicionalmente, se observó que a las $24 \mathrm{~h}$ de fermentación los \% $\mathrm{H}$ disminuyeron significativamente $(P<0.01)$, y a las $48 \mathrm{~h}$ nuevamente aumentaron; se indica también el aumento y posterior disminución $(\mathrm{P}<0.01)$ de \%MS a las 24 y $48 \mathrm{~h}$ de incubación, respectivamente. Se sugiere que la disminución de la MS de los diferentes alimentos al inicio de la fermentación, con respecto a la calculada, está relacionada con la adición de agua al sistema, al diluir la urea $(9,21)$, lo que corresponde a los resultados presentados a 24 h (Tabla III). Así mismo, con el trascurso del tiempo se empieza a liberar mayor cantidad de agua por los procesos metabólicos desarrollados por los microorganismos. Se indica que la acción microbiana provoca una reducción de MS en el tiempo durante procesos FES, producto de la utilización de azúcares (sacarosa, glucosa, fructosa) y almidón en sus procesos metabólicos hidrolíticos, que generan agua, $\mathrm{CO}_{2}$ y AGV, lo que se ha reportado (26) al evaluar procesos FES luego de $48 \mathrm{~h}$ de fermentación.

Parte del agua producida durante la hidrólisis y la oxidación de las moléculas pudiera evaporarse por el calor metabólico que se genera durante el proceso de FES $(18,22,30)$; esto coincide con el comportamiento evaluado en los valores de MS a 48 h de fermentación (19 y 17,9\% a 25 y $30{ }^{\circ} \mathrm{C}$, respectivamente), donde se observa que dichos valores vuelven a ser similares a los de la hora 0 , que también fue tomada con un resultado de1 5.7\%; adicionalmente, se observa que los porcentajes de $\mathrm{N}-\mathrm{NH} 3$ y la cantidad de AGVt también se encuentran muy cercanos a 0 .

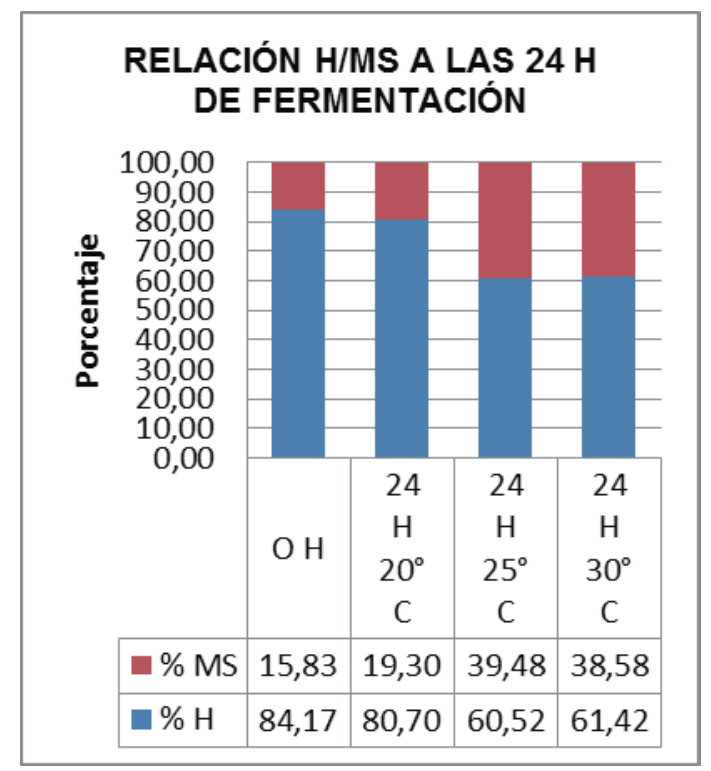

Figura 1. Valores de materia seca y humedad a las 24 h de fermentación.



Figura 2. Valores de materia seca y humedad a las 48 h de fermentación.

En la Figura 1 se puede observar que el producto presentó aumento en el \%MS luego de $24 \mathrm{~h}$ de incubación en las diferentes temperaturas. Por su parte, en la figura 2 el producto mantenido 
a 25 y $30{ }^{\circ} \mathrm{C}$ disminuyó a valores similares al de la hora 0; este comportamiento, como se explicó anteriormente, se puede atribuir a que en el trascurso del tiempo de fermentación se empieza a liberar mayor cantidad de agua, por los procesos metabólicos desarrollados por los microorganismos, que disminuyen el porcentaje de MS.

\section{Conclusiones}

El producto FES-papa incubado a diferente temperatura y tiempo presenta modificaciones de relevancia en indicadores fermentativos y en el contenido de humedad y materia seca. El porcentaje de N-NH3 y de AGVt desciende con el tiempo, lo que se relaciona con el $\mathrm{pH}$. Según el contenido de MS y los indicadores fermentativos, este producto se pudiera emplear en la alimentación animal y contribuir a contrarrestar la contaminación ambiental.

\section{Referencias}

(1) Superintendencia de Industria y Comercio. Cadena productiva de la papa: Diagnostico de libre competencia. 2012. (Acceso: enero 05, 2014). Disponible en: http://www.sic.gov.co/ documents/10157/973ad164-55ea-4c55-9d24$38 f 11403 \mathrm{e} 400$.

(2) Vélez Goyeneche A. Papitas para la China y la India. Revista Papa, 2013, pp. 4-10. Colombia: Fedepapa.

(3) Betancourt JE, Cuastumal HB, Rodríguez SP, Navia JF, Insuasty EG. Alimentación de vacas Holstein con suplemento de papa de desperdicio (Solanum tuberosum) y acacia negra (Acacia decurrens), y su efecto en la calidad de leche. Investig. pecu, 1(2): 41-51, 2012, Pasto, Nariño.

(4) Prada Ospina R. Alternativa de aprovechamiento eficiente de residuos biodegradables: el caso del almidón residual derivado de la industrialización de la papa. Rev. esc.adm.neg, 72(1): 182-192. Bogotá, 2012.

(5) Gualtieri MJ, Villalta C, Diaz LE, Medina G, Lapenna E, Rondon ME. Producción de biomasa Saccharomy cescerevisiae y Candidautilis usando residuos de pulpa de Coffea arabica L. INHRR, 28(2): 13 p. Caracas, 2007.
(6) Siebald E, Goic L, Matzner M. Alimentación de rumiantes con papa de desecho. Boletín Técnico, N. ${ }^{\circ}$ 88, 2002. Instituto de Investigaciones Agropecuarias - Centro Regional de Investigaciones Remehue. (Acceso: Enero 27, 2014). Disponible en: http://www. produccion-animal.com.ar/informacion_tecnica/ suplementacion/99-papa.pdf..

(7) Montoya N, Pino ID, Correa HJ. Evaluación de la suplementación con papa (Solanum tuberosum) durante la lactancia en vacas Holstein. Rev Col CiencPec 17(3):241-249. Medellín, 2004.

(8) Monje A. Estrategias de alimentación para los sistemas de producción de carne vacuna ante situaciones de déficit de forraje. 2006. (Acceso: noviembre 30, 2013). Disponible en: http:// www.elsitioagricola.com/gacetillas/concepcion/ co2006gacetillas/20060602alimentacion.asp.

(1) Elías A, Lezcano O, Lezcano P, Cordero J, Quintana, L. Reseña descriptiva sobre el desarrollo de una tecnología de enriquecimiento proteínico en la caña de azúcar mediante fermentación sólida (Saccharina). Rev. Cubana Cienc. Agríc, 24(1): 3-12.

(10)Conway EJ. Microdiffusión analysis and volumetric error. 4 Ed. Crosby e Ockuldod and Sons, Itd. London. 1967.

(11) Cottyn, B.G \& Boucqué, C.V. Rapid method for gas fluid agric. Fd. Chem. 16: 107. 1968.

(12) AOAC. Oficial methods in analiysis. 16 Ed. Off. agricu.chem., Waschington, D.C, USA. 1995.

(13) SAS. Versión 9.2 para Windows. 2009.

(14)Rodríguez, B.Y. Obtención de un alimento energético proteico a través de la FES de la caña de azúcar y el tubérculo de yuca [Tesis de Maestría]. Universidad Agraria de la Habana. Instituto de Ciencia Animal. Habana, Cuba. 2005.

(15) Elías, A. \& Lezcano, O. Efecto de los niveles de inclusión harina de maíz sobre la fermentación de caña de azúcar. Rev. Cubana Cienc. Agríc, 1994; 28: 319.

(16) Elías, A. \& Lezcano O. Inclusión de niveles de harina de soya desgrasada y sin desgrasar en la 
fermentación de la caña de azúcar en estado sólido. Sacchasoya. Rev. Cubana Cien. Agric., 2000; 34: 143.

(17) Elías A, Lezcano O, Herrera F. "Algunos indicadores bromatológicos y productos finales de la fermentación para la obtención de cuatro tipos de Saccharina inoculados con Vitafert". Rev. Cubana Cienc. Agric., 2001; 35(2): 153158.

(18) Díaz Plascencia D. Desarrollo de un inoculo con diferentes sustratos mediante fermentación solida sumergida. REDVET [en línea]; 12(1). 2011. (Fecha de acceso: 11 de enero 2013). Disponible en: http://www.veterinaria.org/revistas/redvet/ n010111/011101.pdf..

(19) Pandey, A., Soccol C.R., Rodríguez-León, J.A. \&Nigam, P. Solid-state fermentation in biotechnology. Fundamentals and applications. Asiatech Publishers, Inc. New Delhi. 221 p. 2001.

(20) Calderón J, Elías A, Valdivie M. Dinámica de la fermentación en estado sólido de la camas de cascarilla de café en inicio de ponedoras inoculadas con vitafert. REDVET, 2005; 6(5): 8.

(21) Valiño, E., A. Elías, E. Álvarez, E. Regalado y J. Cordero. Dinámica de crecimiento de la microbiota de la caña de azúcar durante la obtención de saccharina. Rev. Cubana de Cienc. Agric., 1992; 26: 297.

(22) Fernández Cabrera CR. Efectos de los niveles de urea en el Sacchapulido sobre los patrones de fermentación ruminal [Tesis de maestría]. Tabasco: Colegio de Postgraduados, Institución de Enseñanza e Investigación en Ciencias Agrícolas. 2009.

(23) Ramos Juárez JA. Obtención de un concentrado energético proteínico por fermentación en estado sólido de la caña de azúcar para bovinos en ceba [Tesis doctoral]. La Habana: Instituto de Ciencia Animal, Departamento de Ciencias Biofisiológicas. 2005.
(24) Álvarez L, Mendoza G, Aranda E, Ramos J, Mora O, Hernández P, García. Efecto del nivel de sustitución del bagacillo de retorno (Pachaquil), fermentado en estado sólido y ensilado, en el comportamiento productivo de vaquillas en el trópico. Rev. Cubana Cienc. Agric., 2011; 45(3): 257-260.

(25) Rodríguez Z, Elías A, Boucourt R, Núñez O. Efectos de los niveles de nitrógeno ureico en la síntesis proteica durante la fermentación de mezclas de caña (Saccharum officinarum) y boniato (Ipomea batata Lam.). Rev. Cubana Cien. Agric., 2001; 35(1): 29-36.

(26) Valiño E., Elías A., Álvarez E. Quintana M. \& Montes de Oca N. Composición de especies de bacterias aisladas del proceso de obtención de la Saccharina. II. Bacterias gram positivas. Rev. Cubana Cienc. Agríc., 1994; 28: 75.

(27) Rodríguez Z, Boucourt R, Elías A, Madera M. Dinámica de fermentación de mezclas de caña (Saccharum officinarum) y boniato (Ipomea batata). Rev. Cubana Cienc. Agric., 2001; 35(2): 147-151.

(28) Rodríguez Z, Boucourt R, Elías A, Herrera F, Núñez O. Efecto del grosor de la capa en la dinámica de fermentación de mezclas de caña (Saccharum officinarum) y boniato (Ipomea batata Lam). Rev. Cubana Cienc. Agric., 2006; 40(2): 173-182.

(29) Fonseca D. Evaluación de una dieta con diferentes porcentajes de inclusión de papa fresca mezclada con un alimento a base de harinas sobre la producción y calidad de leche en vacas Holstein [Tesis de grado]. Facultad de Ciencias Agropecuarias: Universidad Pedagógica y Tecnológica de Colombia. 2013.

(30) Mitchell D.A., Berovic M. \& Krieger N. Overview of solid state bioprocessing. Biotechnology Annual Review. Elsevier Science. Animal Feed Science and Technology. 8:183-200. DOI: 10.1016/S1387-2656(02)08009-2. 2002. 